\title{
Manifesto of computational social science
}

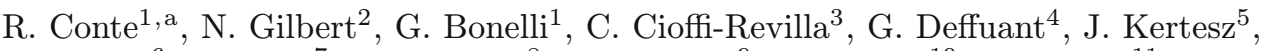 \\ V. Loreto ${ }^{6}$, S. Moat ${ }^{7}$, J.-P. Nadal ${ }^{8}$, A. Sanchez ${ }^{9}$, A. Nowak ${ }^{10}$, A. Flache ${ }^{11}$, \\ M. San Miguel ${ }^{12}$, and D. Helbing ${ }^{13}$ \\ 1 ISTC-CNR, Italy \\ 2 CRESS, University of Surrey, UK \\ 3 Center for Social Complexity, George Mason University, USA \\ ${ }^{4}$ National Research Institute of Science and Technology for Environment and Agriculture \\ (IRSTEA), France \\ ${ }^{5}$ Institute of Physics, Budapest University of Technology and Economics, Hungary \\ 6 Sapienza University of Rome, Italy \\ 7 University College London, UK \\ 8 CNRS, France \\ 9 GISC, Carlos III University of Madrid, Spain \\ 10 Center for Complex Systems, University of Warsaw, Poland \\ 11 ICS, University of Groningen, The Netherlands \\ 12 IFISC (CSIC-UIB), Campus Universitat Illes Balears, 07071 Palma de Mallorca, Spain \\ 13 ETH Zurich, Switzerland
}

Received 1 August 2012 / Received in final form 9 October 2012

Published online 5 December 2012

\begin{abstract}
The increasing integration of technology into our lives has created unprecedented volumes of data on society's everyday behaviour. Such data opens up exciting new opportunities to work towards a quantitative understanding of our complex social systems, within the realms of a new discipline known as Computational Social Science. Against a background of financial crises, riots and international epidemics, the urgent need for a greater comprehension of the complexity of our interconnected global society and an ability to apply such insights in policy decisions is clear. This manifesto outlines the objectives of this new scientific direction, considering the challenges involved in it, and the extensive impact on science, technology and society that the success of this endeavour is likely to bring about.
\end{abstract}

\section{Objectives and opportunities}

In a world of demographic explosion, global crises, ethnic and religious disturbances and increasing crime the understanding of the structure and function of society, as well as the nature of its changes, is crucial for governance and for the well being of people. Humanity is currently facing grand challenges. Setting aside environmental issues and the depletion of natural resources, we have to cope with formidable social and political problems:

\footnotetext{
a e-mail: rosaria.conte@istc.cnr.it
} 
- Change of the population structure (change of birth rate, migration);

- Financial and economic instability (trust, consumption and investments; sovereign debt, taxation, and inflation/deflation; sustainability of social welfare systems, and so on);

- Social, economic and political divide (among people of different gender, age, education, income, religion, culture, language, preferences);

- Threats against health (due to the spreading of epidemics, but also to unhealthy diets and habits);

- Unbalance of power in a multi-polar world;

- Organized crime, including cyber-crime, social unrest and war;

- Uncertainty in institutional design and dynamics (regarding regulations, authority, corruption, balance between global and local, central and decentralized systems);

- Unethical usage of communication and information systems (cyber risks, violation of privacy, misuse of sensitive data, spam).

In the last couple of years, social scientists have started to organize and classify the number, variety, and severity of criticalities, if not pathologies and failures, recurring in complex social systems $[1,2]$. These are amongst the most severe social problems, difficult to predict and treat, and raising serious social alarm.

Furthermore, human society has never before changed as fast as it is changing today. Technological development has opened entirely new channels of communication, induced new behavioural patterns, substantially influenced organization principles, and its products are becoming history-forming factors. We human beings have preserved our basic, genetically determined biological properties over tens of thousands of years but our social behaviour seems to be altered with an unprecedented speed, continuously challenging our adaptivity.

Part of the difficulty for us to respond to the challenges mentioned above is inherent to fundamental features of social complexity. Complex social systems are characterised by multiple ontological levels with multidirectional connections, proceeding not only from the micro to the macroscopic levels but also back from the macro to the micro-levels [3]. Furthermore, complex social systems present a far-reaching and accelerated diffusion of phenomena, behaviours and cultural traits. Accelerated contagion leads on one hand to new systems' properties emerging at the aggregate level - for example new public opinions and political movements, new global and local identities, collective preferences, attitudes, even moods, etc. - and on the other to major critical event in the social economic and/or political spheres, such as global financial crises and the collapse of regimes.

Finally, complex social systems do often show interdependences and interferences among their properties and processes of transformation. The interplay between cultural and biological evolution shows unexpected intricacies, far from the parallel predicted by the Dual Inheritance Theory [4], as shown by the Demographic Transition (DT) model [5]. Based on an interpretation of demographic history developed in 1929 by the American demographer Warren Thompson, the DT model points to a growing gap between economic and demographic growth: all over the world, the higher the average income of the population the lower its birth rate.

These problems depend on the same circumstances that might help us find solutions: a high degree of poorly understood and poorly investigated technology-driven innovation. ICT applications seem to act both in favour and against our capacity to answer the grand challenges before us. The widespread access to the Internet is seriously and often positively impacting the frequency, range and style of human communication and interaction, leading to heterogeneous interconnected networks. Electronic communications seem to have played a fundamental role in the diffusion and organization of the protest movements arising in Northern Africa, and leading to regime change. At the same time, the view that social networks connect people is 
oversimplified, and the question remains open as to what types of connections are established among them, whether, for example, pro or antisocial, and - since resources like time are limited - to the expense of what and of whom. The alternative offered by Internet to the hierarchical organization of cultural production and specialised professional advice pushes up symmetrical, horizontal interactions. At the same time, the Open Source community challenges the foundation of intellectual property as well as the institution of truth certification. Are these effects only signs of providential progress? What about the nature and functioning of economic, cultural and political institutions? What about the credibility of the information spread and its effective truth-value?

That much for the negative side of the coin. But there is also a positive side. Information and communication technologies can greatly enhance the possibility to uncover the laws of the society. First, ICT produces a flood of data. These data represent traces of almost all kinds of activities of individuals enabling an entirely new scientific approach for social analysis. Second, the development of computer capacities makes it possible to handle the data deluge and to invent models that reflect the diversity and complexity of the society.

The analysis of huge data sets as obtained, say, from mobile phone calls, social networks, or commercial activities provides insight into phenomena and processes at the societal level. Investigating peoples' electronic footprints did already contribute to understand the relationship between the structure of the society and the intensity of relationships [6] and the way pandemic diseases spread [7], as well as to identify the main laws of human communication behaviour [8].

The traditional tools of social sciences would at most scratch the surface of these issues, whereas new tools can shed light onto social behaviour from totally different angles. Possibilities ranging from supercomputers to distributed computing make the execution of large-scale, heterogeneous multi-agent programs possible, programs which prove particularly apt to model the complexity of social and behavioural systems.

The new ICT-enabled study of society has been named computational social science [9]. This is a truly interdisciplinary approach, where social and behavioural scientists, cognitive scientists, agent theorists, computer scientists, mathematicians and physicists cooperate side-by-side to come up with innovative and theory-grounded models of the target phenomena. Computational social scientists strongly believe that a new era has started in the understanding of the structure and function of our society at different levels [9].

On the one hand, computational social science is aimed to favour and take advantage of massive ICT data. On the other, it is a model-based science yielding both predictive and explanatory models. Hence, it is intended to profit from the modelling instruments made available by ICT for producing generative models of large-scale multi-agent systems. Both objectives must be achieved to turn social science into applicable tools that can inform decision makers about issues of major concern.

In this paper, we will work towards the drafting of a Manifesto for the new Computational Social Science.

The paper will unfold as follows: in the next section, the state of the art of the field will be discussed. In the third section, its main characteristics will be examined. In the fourth section, the main challenges the new field is facing will be addressed. Finally, in the fifth section, we will turn our attention to compare and discuss the types of models that are compatible or necessary for a computational social scientific program as outlined in the previous part of the paper. Final considerations will conclude the paper, but not the process to constitute the new discipline, that for a Computational Social Science is an inherently dynamic scientific program. This paper is only part of the beginning of it. 


\section{State of the art}

\subsection{Emergent phenomena at the aggregate level}

The computational study of social phenomena has been focused on the emergence of all sorts of collective phenomena and behaviours from among individual systems in interaction - including segregation [10], cooperation [11], reciprocity [13], social norms [14-17], institutions [18], etc. Let us briefly re-examine the major research directions.

Emergent social behaviour. The study of emergent social behaviour did greatly benefit from computational and simulation-based modelling. Although even phenomena like civil violence and rebellion have been investigated [19], computational modelling has so far been mainly applied to behaviours like altruism, cooperation and norm conformity.

The study of altruism has generally been cast into the evolutionary framework. However, computational studies of the cultural dimension of this phenomenon exist - see the economy of $[20,21]$; cf. later on in the paper, 4.1.3.

While the perspective in which the study of cooperation is generally framed is game theoretic, the current frontiers in the computational study of cooperation arise in other formal fields, like complex systems science. Socio-semantic systems dragged the attention of the scientific community to investigate quantitatively how cooperative phenomena emerge and can be harnessed to improve the performance of collective tasks [22-24].

The most insightful computational studies of altruism are due to Nowak and Sigmund Nowak and Sigmund 1998, who had the merit, among others, to point out the role of image scoring in the evolution of donation. In turn, image scoring gave impulse to the study of reputation (for two recent reviews see Walker [26] and Ebbers and Wijnberg [27] in the emergence of cooperation from repeated interaction (the so called shadow of the future, [28]) and from networks of interconnected agents [29].

Thanks to its evolutionary background, the computational modelling of prosocial behaviour was aimed to identify the distal causes of altruism and cooperation, i.e., their impact on the individual and the social group's chances for survival. One of the principal directions of investigation of the distal causes of cooperation concerned strong reciprocity, i.e., the spontaneous attitude to punish free-riders. Evolutionary game theorists showed the positive effect of strong reciprocity on both the cooperators' and the groups' fitness by simulating artificial hunter-gatherers populations. The missed point in the evolutionary study of prosocial behaviour is the proximate causes, i.e., the behavioural and mental mechanisms on which reciprocity is implemented. Evolutionary theories point to the competitive advantage (distal cause) of the behaviours to explain, leaving aside the question of how they could have ever appeared in the behavioural repertoire of a species (proximate cause). The application of computational methodologies to study also the proximate causes represents a major challenge for the future computational social science.

Emergent social aggregates. The best known and most influential work in this area is Schelling's seminal paper on segregation [10], which gave impulse to a great deal of computational studies of emergent structures at the aggregate level (see e.g. [30-32]). Computational studies of spontaneous group formation and the emergence of coalitions and collective entities are also at study among social scientists and game theorists since long [33]. This research direction had the merit to point out the role of extortion and tribute as mechanisms of political coalition formation.

However, no much attention was given to the opposite direction of influence, i.e., downward causation or second order emergence [34,35]. Furthermore, the study of emerging phenomena at the aggregate level not always shed light on the foundations of social structures. Social networks, for example, are investigated by the properties they 
exhibit once they have emerged (e.g., scale-freeness). Instead, poor attention has been paid so far to the conditions favouring, and allowing to predict, the emergence of social networks (see [36] and later on in this paper, 4.1.2). Agent-based modellers have begun to address this issue and to study the link between fundamental behavioural processes, social conditions, and the macroscopic structure of emergent complex networks [37].

Emergent institutions. While the study of emergent social behaviour has mainly addressed positive social action, a wide spectrum of institutions has been observed to emerge in computational environments, from the market [38] to money [39]; from social organization [40] to the modern state [41].

As to theoretical frameworks, the study of emerging conventions and social norms $[14,19,42-44]$ is greatly indebted to the game theoretic framework and to the philosophical bases of the rationality theory $[16,45]$. Nonetheless, agent-based models of norm emergence have been developed by authors not committed to a rational view of agency [46-48]. The study of norm emergence turned out to be one of the most prolific domains of investigation of computational social science. Nonetheless, a number of questions have been left open, the most important of which perhaps is how to account for norm compliance.

In sum, the study of emergent phenomena has largely profited from the adoption of computational methodologies. Rather than conclusive, however, the results obtained so far indicate new promising directions of research, in particular, (a) the micro-foundations of social structures and networks; (b) the proximate causes in evolutionary explanation, i.e., the internal mechanisms that contribute to explain the advantages of the target behaviours for the individual and for the group and (c) the way back in the dynamics of emergent phenomena like norms and institutions, and how they manage to be adopted by executors. These are all important challenges for a field, like computational social science, that has the potential to re-found the science of society.

\subsection{Social learning systems and mechanisms}

Rather than a topic of investigation per se, learning is a property on which a great deal of computational social science builds upon (see N 4.5 Democratising Big Data, Complexity Modelling and Collective Intelligence). All sorts of social dynamics are attributed to learning processes, based on direct reinforcement or imitation. Replicator dynamics has been strongly influential in the study of social and economic processes, and still is one of the techniques on which computational models of social dynamics are implemented.

Despite the conceptual and theoretical weaknesses of the models and techniques used, learning systems have had a strongly innovative effect on the study of social influence, yielding some of the most brilliant results ever achieved by computational social science so far. One example is the out-of-equilibrium economy (see [49]; see also the non-equilibrium social science of Hales and Johnson, in which what matters is not the equilibrium obtained, but how it is obtained. Learning is a fundamental mechanism that may lead, but not necessarily, to achieve an equilibrium under specific conditions. As shown by Arthur's agent-based model of an artificial rudimentary stock market, the possibility to achieve an equilibrium depends on the speed of the learning process: the faster the process, the more unstable the equilibrium. Under special conditions, the non-linear and inconsistent effects of learning become paradoxical: for instance, in the minority game [50], people adjust their behaviour to their own expectations concerning certain events. However, while adjusting their behaviour, people modify the expected conditions, somehow contributing to disconfirm their expectations. This problem, which has received a great deal of attention in the last 
couple of decades, shows the paradoxical effects of learning and the complex, out-ofequilibrium character of complex problems. Further computational work has revealed how learning dynamics can lead to solutions of the problem of cooperation that are out-of-equilibrium from a rational actor perspective, but may be robust when agents are boundedly rational [51]. The non-equilibrium phenomena generated by learning dynamics are a decisive battlefield for computational social science.

\subsection{Quantitative computational social science}

Computational social science, in terms of agent-based models (ABM), has existed for a few years. To date, it has been used more as a qualitative tool, e.g., to provide plausible explanations to social phenomena [52]. However, much effort has recently been made towards achieving a more quantitative orientation of this kind of research, and advances have been made in several important directions.

One important topic in this line of research is the assessment of the validity of simulations: As Gilbert [34] put it:

"You should assume that, no matter how carefully you have designed and built your simulation, it will contain bugs (code that does something different to what you wanted and expected)."

Techniques to verify the correctness of the code have been developed and are increasingly used [53] including, for instance, comparison of simplified versions of the model with analytical results or duplication of simulations in different machines, languages or both. Less progress has been made, though, towards simulations that can be quantitatively compared with specific social phenomena, and validation (i.e., assessing the validity of a model by the extent that it provides a satisfactory range of accuracy consistent with its intended application) is often only qualitative. Quantitative validation will require detailed explorations of the parameter space that, in turn, would call for appropriate computational facilities, such as grid computing; these would bring back the issue of code verification. Suitable quantities for meaningful comparisons need to be identified and measured in the social context of interest (or at least in controlled experiments). These are crucial steps that need to be carefully addressed if computational science, and in particular model simulation, are to be really quantitative.

A second relevant problem with which computational social science is dealing in its progress towards becoming quantitative is massive data analysis. In addition to the computational requirements, common to all the questions mentioned in this section, the design and implementation of efficient and reliable analysis algorithms is at the core of the research efforts these days. Examples like the controversy on reports of contagion of obesity in social networks $[54,55]$ clearly show the importance of rigor in analyzing data in a meaningful way, allowing to distinguish between factors leading to certain behaviors and to identify causation when possible. Important progress has here been made by methods that tackle the problem of statistically modeling complex and interdependent dynamics of behavior and networks with integrating agent-based simulation techniques with statistical approaches for parameter selection [56]. The further development of these approaches becomes even more acute when data are so massive as to prevent the use of well-known algorithms, something that is become more and more frequent as new sources of data become available.

Finally and importantly, the previous topic is certainly related with data driven simulations, carried out to compare with, understand, and if possible predict real-life phenomena. Simulation models such as ABMs can be constructed to support persistent run-time interactions between computer agents and real-world entities via general 
types of input-output data streams [57]. In this way, ABMs become data-driven dynamic applications systems [58] entailing the ability to incorporate additional data into an executable application and, vice versa, the ability of applications to dynamically steer the measurement process. Again, the computational and ICT requirements to augment this kind of approach with massive sources of data to simulate large social groups are enormous, and research along this line is at the forefront of the field right now.

\section{Innovative approach: How to characterise the field of computational social science}

Are we well equipped to tackle the BigProblems mentioned above? Undoubtedly, we are developing valuable instruments and techniques for generating, gathering, and analysing data about grand challenges, but how about BigThinking, grand theories matching grand challenges? Theories grow slowly, impeded by entrenched assumptions and lack of data. The large-scale, founding constructs that should drive our understanding of society are debated and misunderstood. In social sciences, there is no consensus on the general mechanisms that underlie phenomena like institutions, norm compliance and enforcement, reputation, trust, etc. Even cooperation, one of the most studied aspects of social interaction, is still locked between free riding and punishment. With Putterman [11], we believe that important social dilemmas can only be solved if:

"The human sociality that evolved in our small-group past is robust enough to overcome the ever-present temptations to free ride".

How can we understand and help manage complex social systems if we still do not understand the basics of sociality? Is the new world of automated information treatment going to provide any help?

The answer is: yes, ICT can provide significant help for social science. Not only ICT can help access, analyse and build upon BigData, i.e. new type of massive data, for addressing BigProblems. It can also help provide instruments for BigThinking. Indeed, computational social science can be characterised along two main aspects, which both take advantage, one way or the other, from ICT developments: a) BigData, and $b$ ) the role of computation in inspiring, formalizing and implementing the core scientific concepts, principles, and ideas of computational social science.

\subsection{BigData}

Computational social science is intended to process data and run simulations at planetary scale, where up to the whole world population is considered, in order to get a better understanding of global social dynamics. This makes sense in a more and more interconnected world, where the events occurring in one place can have tremendous consequences on the other side of the globe [12]. For instance, migrations, the diffusion of diseases, the consumption or production of goods should now all be considered at planetary scale and involve the whole world population.

The latest evolution of Information and Communication Technologies (ICT) has increasingly concerned the inclusion of users in the production of information. Nowadays, users are not only able to exchange messages, images and sounds with other individual peers, but also with whole communities whose size and composition can be defined and tuned by the user him/herself. Moreover, the digital paradigm has allowed the integration of multiple information and communication sources, including connected PCs, phones and cameras. Accordingly, the distinction between consumers 
and producers of information, typical of a past era dominated by newspapers and television, is vanishing.

Such an interconnected communication network has dramatically enlarged the access to information sources with an undeniable advantage for the citizenship. At the same time, it is presenting new challenges. Information broadcast by uncontrolled sources could overload the network with noisy signals, preventing the meaningful ones from being received by the desirable recipients. As a consequence, users' attention could be exhausted by useless information.

To overcome obstacles to the use of this increased amount of data, a number of technologies have been developed. More sophisticated communication platforms have emerged, up to current Web 2.0 social networks accessible from PCs and cell phones where users can collectively categorize and evaluate the content they browse, providing the community with an efficient information filter. The classification of digital resources is typically performed by assigning labels (or tags) or scores to resources. This collaborative categorization has given birth to several web-based folksonomies. Consequently, the most popular websites now incorporate some sort of collaborative categorization tools.

This ICT infrastructure has been applied not only to favour data exchange among people, but also to outsource productive tasks. The main difference between this form of crowdsourcing and traditional labour markets lies in the absence of prearranged duties that workers deal with an idiosyncratic effort, while the infrastructure takes care of summing up all contributions despite the heterogeneity and number of users. First examples have concerned highly specialized tasks, such as open source software development or scientific programs [59] that could be broken into smaller operations performed by uncoordinated volunteers. More recently, the range of activities being crowdsourced has expanded and forms a world-wide labour market facing tasks proposed by various agencies and companies asking, e.g., for technological and marketing solutions, or by research groups looking for volunteers for test and data mining activities $[60,61]$. These kinds of infrastructures, therefore, are particularly appropriate for the involvement of citizens in distributed sensing experiments $[62,63]$.

Devices employed to get connected to communication networks have converged in size and technological standards, expanding more and more the availability of an Internet connection throughout daily life. Thus, users can now easily form dedicated networks providing data that monitor particular issues. Such sensing networks can be of an opportunistic or participatory type.

The participation of users in the monitoring affects both the resolution and the quality of the data collected. Traditional sensing generally involves a small number of highly controlled observation points. The low spatial resolution of the data gathered in this way is compensated by the high data quality certified by the controlling agency. On the other hand, distributed sensing relies on the possibility of gathering large amounts of data from many uncontrolled sources, which cannot ensure high data quality standards; however, by means of statistical methods together with the possibility of storing and post-processing large datasets, this quality gap with respect to traditional sensing can be overcome. Reasonably, users provide larger quantities of data if the observed phenomenon and its management directly concern the community involved in participatory sensing experiments. For example, people might be interested in reporting meteorological observations in order to improve existing models and receive more accurate weather forecasts, and this, as a virtuous feedback, could be a reason for a citizen to provide more data to meteorological centres.

For all these reasons, the application of a novel ICT-based sensing framework may have a stronger impact here than in other areas, since the knowledge of the underlying social interaction is crucial from many points of view: the quality of the 
environment is strongly affected by the behaviour of individuals in their most ordinary daily situations; citizens' behaviour, in turn, depends on their awareness; many bad environmental practices arise when citizens do not coordinate in order to attain a global optimal usage of collective resources, but rather pursue their own profit selfishly - resulting in an even worse long term individual performance.

Hence, the field of computational social science is characterised as a new field of science in which new type of data, largely made available by new ICT applications, can be used to produce large-scale computational models of social phenomena. However, the new field is not only characterised by new data at higher levels of temporal and spatial scale, but also by new principles and concepts.

\subsection{Core scientific concepts and ideas}

Computational social science is a powerful tool for fostering our understanding of the complexities of real socio-economic systems, by building "virtual computational social worlds" that we can analyze, experiment with, feed with and test against empirical data on a hitherto unprecedented scale. A range of excellent papers has been written to make this point (e.g., [9]).

Computational models provide quantitative and qualitative models of social phenomena. One critical application is generative explanations (see 4.4.2), in the form of computer code, that reproduce some key features of societies. Hence, agent-based modelling (multi-agent systems) plays a central role in computational social science, because people (i.e., agents) are the primary subjects of social theories. Social science is about how people think (psychology), handle wealth (economics), relate to each other (sociology), govern themselves (political science), and create culture (anthropology). Agentification is the process of formalizing a social theory as an agent-based model.

The mind - beliefs, desires, intentions, values, and their processes - is at the root of human social complexity. Cognitive science and social psychology are both necessary for computational social science. Agent-based models benefit from richer cognitive architectures, depending on specific modelling goals. For a specific discussion on this see Sect. 4.

Computational social science is applied to real-world societies. These must be complex or could not exist, because a set of critical functions is necessary for every community to operate and endure. Computational social science is aimed to pay attention not only and not primarily to variables and equations, but to the entities the social world consists primarily of, i.e., people, ideas, human-made artefacts, and their relations within ecosystems. These entities are modelled as computational objects that encapsulate attributes and dynamics.

Another crucial idea of computational social science is adaptation. Social complexity results from human adaptation to challenging environments (Simon's Principle). Social complexity as an emergent phenomenon is caused by successful adaptation. Coupled socio-natural and socio-technical systems are typical examples of complex adaptation.

Social complexity can be caused by uncertainty, which is commonly misunderstood as something that cannot be known. We need to understand uncertainty and related ideas (e.g., inequality, entropy). Probability and other scientific theories of uncertainty (for example theory-driven models of opinion formation, revision and dynamics in interplay with other mental constructs, like beliefs) are essential for understanding social complexity.

The possibility of social change is ubiquitous and important in social life. Nothing social occurs "out of the blue," without antecedents. What may happen is as important for social complexity as what has happened. 
Scaling is another crucial aspect of computational social science. The scaling typical of a power law ("80-20 Rule") follows a pattern of "many-some-rare". Income, wealth, conflicts, organizations, cities, and other features of social life obey power laws. The first discoveries of power laws occurred in social science, not physics. Computational social theories and agent-based models are capable of producing power laws and other real-world social distributions. Not all scaling is the same; some cases are special because of their criticality. Social scientists were not the first to recognize criticality; physicists did. Criticality is insightful for some social phenomena (e.g., conflict), but still puzzling for others.

One of the most interesting aspects of social life is out-of-equilibrium. Most social distributions are not Gaussian, or bell-shaped. They are often heavy-tailed, powerlaw (Pareto), Weibull (exponential, Rayleigh, others), log-normal. Natural raw data reflects the typical disequilibrium of social complexity - "normalizing" data using atheoretical transformations (for regression analysis) may destroy valuable information about generative processes. Networks account for much of social complexity. One of the problems is to understand different properties of networks that determine their emergence (see 3.1.2). Social network analysis (SNA) investigates systems of social relations, from cognition to the global system. Computational social science allies of SNA include agent-based modelling, complexity models, and visualization analytics.

Future research directions must include maturation of existing knowledge - both deeper and more interdisciplinary - as well as new knowledge creation through computational methods (visualization analytics). Viable social theories should be agentized, analyzed, and tested with empirical data - from human cognition to international relations, including fields beyond the five social sciences (linguistics, geography, organization science, history, communication, law, and others). The complex triadic nexus among social, artefactual, and natural systems requires computational investigation within an overall science of complexity, drawing upon existing and new methods from the social, behavioural, natural, mathematical, and computational sciences.

\section{Challenges}

Computational social science must be set to answer a number of fundamental scientific questions, perceived from within the scientific community as pivotal to address and help manage the BigProblems of society.

\subsection{Understand levels and directions of interaction}

Levels and directions of interaction are major sources of social complexity. Real-world societies imply several levels of complexity, which are not reduced to the micro and the macro levels but include intermediate levels (groups, tribes, networks, communities, etc.).

Entities belonging to any of these levels interact with one another: individuals interact with other individuals, groups may conflict with other groups, macro-level entities need to coordinate with one another (one Ministry with another, the Parliament with the Government, etc.). But they also interact with entities at any other levels: group members interact with group artefacts, for example norms, customs, and habits, and with macro-entities, like justice courts, fiscal agencies, the electoral system, etc.

Social levels emerge from one another, and retroact on one another. We need to understand how new levels occur, e.g. how groups and coalitions as well as social institutions and other macro-entities are formed. But we also need to understand how these retroact on the produced levels and modify them. 


\subsubsection{A new view on emergence}

Emergence is much studied, but this does not mean it is well understood. Insufficient understanding of emergence phenomena depend on several factors: a) insufficient analysis of the emergence process, b) inadequate models of the micro-level interacting units from which emergence is supposed to proceed, c) unsatisfactory account of the coupling of emergence and downward causation: many social phenomena and entities emerge while at the same time retroact on the lower levels, and it is not possible to account for one direction of the dynamics without considering the complementary one. This is the case with both social artefacts, like institutions, and cultural artefacts, like technologies.

Here we address the first factor, i.e., the necessity for a more detailed and explicit analysis of the emergence process. Do we really need one? What are the problems that emergence helps us address? In the next subsection, the second factor (groups and networks) will be addressed (4.1.2). The third factor of inadequacy of existing models of emergence will be treated as the Micro-Macro link problem (4.1.3).

What is meant by emergence, and why do we need such a notion? Let us start by a definition of social emergent processes [64] as phenomena a) for which the conditions for its occurence are well defined, b) non-deliberately but spontaneously, c) modifying the entities involved, in particular, interfering with their fates but unlike learning, without modifying the internal states of the producing entities, and d) unlike evolutionary effects, non-transmissible. This definition prevents emergence from collapsing on the notion of "yet unpredicted events" and allows us to tackle systematic but nondeliberate processes of social influence that abound in social life and are responsible for a number of critical social phenomena, from the self-fulfilling prophecy, to social facilitation, social inertia, stalemates etc. A theoretical understanding of emergence is particularly important as it often produces paradoxical phenomena where individual intentions produce unexpected aggregate results with potentially disastrous consequences, as in rational herding, free-rider behavior, or unintended ethnic segregation. Typically one expects an emergent social phenomenon to be characterized by an abrupt collective change that can be well described, in some parameter space, either in the language of dynamical systems as a bifurcation, or, in the language of statistical physics as a phase transition.

Emergence is usually seen as a bottom-up process but horizontal emergence occurs as well. One example is the self-fulfilling prophecy, which is addressed by a great deal of empirical social psychological studies. In general, a self-fulfilling prophecy occurs when individuals gradually and non-deliberately tend to assume properties and behaviours corresponding to the expectations of those with whom they interact. One interesting question concerns the dual of the self-fulfilling prophecy: it is unclear whether the mechanism of self-defeating prophecy exists. Finally, a question still open is whether and under which conditions it is possible to prevent this effect from taking place.

\subsubsection{Networks and group formation}

According to the results of the Harvard Symposium on hard social problems in 2010, one of the top-ten problems is how to achieve good collective behaviour. Unfortunately, a precondition for developing such a theory, namely a model of individual behaviour is missing. Rationality theory is now deemed to be insufficient. Can we model emergent collective behaviour without grounding it on a more plausible model of individual behaviour than is provided by rationality theory?

Two factors prevent modelling the conditions that favour the emergence and prediction of social networks (cf. back to 2.1). First, connections are not allowed to 
emerge from objective relationships, but are either given for granted or created from agents' decisions (an example of the latter is the emergence of role differentiation, [65]). Second, nodes are not modelled as pre-existing entities (agents). Consequently, it is not possible to predict when a set of entities will generate a network (an exchange network, a cooperation network, a trust network, a dependence network, etc.). Within the domain of multiagent systems, based on a more complex view of agency and richer semantics of their relationships, special type of networks have been shown to emerge from among heterogeneous agents, characterised by different mental states and action capacities $[66,67]$. Interestingly, such networks have been shown to allow for different types of positive behaviour - one-shot cooperation and exchange - to be predicted [68]. A system for calculating emergent dependence networks found interesting applications in organizational design and optimization.

One of the tasks of computational social science is to integrate different inputs from adjacent fields in a general theory of social networks that accounts for the properties of existing networks and enables to predict the emergence of new networks.

\subsubsection{Multi-level interactions: The Micro-Macro link}

The interconnection among different levels of social phenomena [69,70], cannot be fully accounted for unless multidirectional processes are modelled, including the downward process from higher-level properties to lower level entities, called Micro-Macro link. More precisely, the Micro-Macro link (see for a recent collection, $[71,72]$ ) is the loop process by which behaviour at the individual level generates higher-level structures (bottom-up process), which feedback to the lower level (top-down), sometimes reinforcing the producing behaviour either directly or indirectly [35].

The Micro-Macro link represents a challenge for our new field of science. How to characterise the whole dynamics? What type of feedback loops does it include? When and how does a given macro-effect retroact on the lower level entities, giving rise to the so-called downward causation? Downward causation is a poorly investigated process, which appears to play a decisive role in many social phenomena. While an emergent effect is always implemented upon interacting micro-social entities, it is not necessarily retroacting on them. When does this happen? When is the MicroMacro circuit closed? Can we predict the occurrence of a micro-macro loop? Can we forecast when a certain emergent effect is likely to be reproduced, and what are the intermediate, or proximate, behavioural or mental causes of its reproduction? Computational social science is expected to address these questions, not before having developed an explicit theory of the Micro-Macro link.

\subsubsection{Interaction with institutions and compliance}

A serious social failure is the breakdown of institutional responsibility that might be expected to follow from the P2P-driven revival of the Wisdom-of-Crowds culture. As the production of knowledge becomes decentralised, no definite locus of responsibility can be traced back and be accountable for the consequences of information spreading. No matter how frequently Wikipedia is false, and even if it is less frequently so than the Stanford Encyclopaedia of Philosophy, who might be blamed and asked to respond for the false news published within it? Should we expect an increasing social uncertainty to follow, especially as people feel unable to claim repair for the damages suffered in consequence of wrong information spreading? In the last couple of decades, we got ourselves busy with questions concerning the impact of ICT on lifestyles, interaction patterns, and thought facilitation or impairment. Poor if any attention 
was paid instead to the way and the extent to which ICT affects the societal artefacts that govern our social lives, and in particular social institutions, and the related degree of social trust. Low compliance is known to be one of the main consequences of the breakdown of institutional responsibility and social trust [73-75]. These issues need to be addressed by Computational Social Science.

\subsection{Modelling culture}

The pioneering computational work by Robert Axelrod [76] addressed the problem of cultural dynamics considering the following question: "if people tend to become more alike in their beliefs, attitudes and behavior when they interact, why do not all differences eventually disappear?" He proposed a simple model to explore competition between globalization and the persistence of cultural diversity. Culture is defined as a set of individual attributes subject to social influence. The model implements a mechanism of homophilic interactions and illustrates how an interaction mechanism of local convergence can generate global polarization (persistence of cultural diversity). However, culturally polarized states have been shown to be unstable against cultural drift in a fixed social network. Computational modeling has identified new mechanisms and conditions that can stabilize the persistence of cultural diversity. Cultural diversity can be understood as a consequence of co-evolving dynamics of agents and network [77]. The social network evolves in tandem with the collective action it makes possible - circumstance makes men as much as men make circumstance. Cultural diversity is also stabilized when cultural influence is not just exerted within interpersonal interactions, but when it is rooted in social pressures from local majorities in actors networks [78]. An additional aspect is that, perhaps surprisingly, a strong mass media message is known to lead to social polarization, while mass media is efficient in producing cultural homogeneity in conditions of weak (and local) broadcast of a message (the power of being subtle, [79]). On the other hand, social interactions can lead to a cultural globalization in a direction different from the one broadcasted by mass media, provided that there are long range links in the network of interactions. Long range links make it possible for collective self-organization to defeat external messages. An important challenge is the understanding of the effects of the new ICT mechanisms of spreading and aggregating information, replacing traditional mass media, on the polarization-globalization issue: The concept of local social circle has been changed, and people are no longer passive receivers of information but sources of information immediately available in a global scale, and simultaneously active players in searching and selecting from a large and distributed number of information sources.

While Axelrod's model considers several cultural features at the same level, other computational studies of cultural dynamics isolate a single cultural aspect such as language [80]. An open question from the standpoint of computational social sciences is the development of integrative models that incorporate different cultural features with different dynamical processes at different scales. Hierarchical and multilevel cultural models are needed to take into account the interdependence of cultural features and the interconnection of cultural dynamics with other social processes.

\subsection{Cross-methodological research}

Well designed experiments constitute another key ingredient of the advancement of science. In the case of the social sciences, they will prove invaluable when combined with simulation work [81]. However, when thinking of large-scale simulations and 
using similarly large-scale data, computational social science is expected to face several crucial challenges for new experimental work in integration with the modelling process:

Design of experiments to test inferences from data. Careful analysis of data should yield intuitions on human interactions and decision-making, but different sets of data or even different analysis of the same data may lead to incompatible proposals. Experimental work specifically designed to discriminate between alternatives should allow choosing among them.

Design of experiments to test simulation predictions, both local and global. Another use of experiments relates to the model or models themselves. These models should lead to predictions both at the micro and at the macro level, possibly involving the corresponding loop. Validation of the models requires more than comparison with available data: Models should offer insights which are experimentally testable. Note also that this is something like a second-order test of conclusions obtained on data.

Protocols for large-scale experiments: there is a need for virtual labs with repeatable procedures and controlled environments. however, going beyond working with dozens of subjects is by no means trivial. For instance, work on experimental game theory by Grujic et al. [82], showed that handling 169 individuals simultaneously is extremely difficult, and this is regarded to be close to what can be done. The design of large-scale (matching that of the data as far as possible) laboratory experiments aimed at identifying local and global effects presents a unique set of challenges, whose resolution requires the development of a commonly agreed methodology and accepted set of tools, possibly including a virtual laboratory and protocols as to how to handle large numbers of volunteers interacting through ICT media (not to be confused with experiments on virtual worlds, [83]).

A word is in order regarding the types of questions such experiments could address. Aside from human behaviour in a fixed social context, in particular decision making in contexts like trading, cooperating or coordinating, or the problems of information aggregation, i.e., how individuals learn and adapt from their social circle, which are of course interesting aspects, experiments should address the formation of the social interaction framework (be it a network or otherwise). This poses additional problems for the experimental design in so far as it may need to be prepared in such a way that it can alter its own context. How to do this in a controllable manner is far from clear, more so if one wants to make sure that the experiment addresses the question one is interested in.

\subsection{How much complexity of agents is needed to get a good picture?}

The discussion so far points to a number of objectives that require higher-level complexity than is allowed by ordinary agent based models of social processes.

First, more mental complexity must be modelled to understand what are the specific mental properties allowing social complexity to be managed and simplified. For example, what are the specific properties or mental attitudes required to cooperate and coordinate with others and give help without aiming to obtain benefits in return? What is needed for people to be willing to participate in social control, thus leading to more distributed costs of norm enforcement?

Second, social intelligence makes society work better by creating social artefacts, both material and immaterial (institutions). Of course, properties of social artefacts are not necessarily linked to individual properties. Therefore the understanding of the individual is not always needed to understand them. However, social artefacts share properties of human producers and users (e.g., limited autonomy, see [84]). If we do 
not understand these properties at the level of human producers and users, we will not be able to understand them at the level of social artefacts.

Third, the most intelligent use of social power is often not signalled, and not easily reconstructed. Social intelligence is more than the capacity to represent and understand the environment. It includes the capacity to represent others' mental representations [85], to reason and manipulate these representations and to actively influence them. Indeed, social power ranges from the exercise of physical strength, to more subtle and insidious manipulative actions in which humans attempt to modify one another's mental states in order to modify one another's future behaviour. The manipulative exercise of power consists of keeping one's intentions hidden to the target. Consequently, social power, at least its manipulative use, is not signalled. To understand and predict these fairly complex mechanisms of power, a relatively complex model of the mind is needed.

Fourth, and consequent, a number of important social phenomena cannot be understood nor fully taken advantage of, without a more complex model of social intelligence and social influence. One example is reputation [86-88]. Reputational systems exist in natural societies since the dawn of mankind. They gave inspiration to a special technology of reputation extensively exploited in commercial applications on the Internet. Cross-methodological evidence [89] shows that the inadequate or unsatisfactory results of the technology of reputation are due to an insufficient understanding of how reputation systems work in natural societies. Analogous considerations apply to the case of trust [90].

Fifth, policy modelling often necessitates to investigate the mental mechanisms which are involved in the behaviours to be modified or strengthened. Consider the role that overconfidence, according to some economists [91], has played in the last financial crisis. How to modify such an attitude? Would the recommendation to not trust banks too much do? And what about bad habits, like drinking, smoking, etc.? Which policies are more likely to succeed in informing diabetics about the benefits to quit unhealthy diets, or young people avoid unsafe or irresponsible behaviours?

\subsection{Towards a new epistemological foundation: What kind of models do we need?}

\subsubsection{Understanding and predicting}

Models are often used to make predictions, a practice sometimes called forecasting. In this case, models represent the properties of actual target systems so that we can predict what these systems will do in the future. The models used for predictive purposes often tell us something about mechanisms. They are output-oriented since, for a given set of initial conditions, they should tell us how the state of the system will evolve in time.

Models can also be used to explain the behaviour or properties of target systems. Explanation is a highly controversial notion [92]. Consistent with the scientific literature on modelling, we hold a view of explaining as "showing how things work" [93]. When we build models for explanatory purposes, we try to make adequate representations of target systems, similar to the predictive case. However, because there is a difference in what we use the model for, different properties enable models to be adequate to their intended use. Unlike predictive uses, which primarily involve optimizing the models to make their output as accurate and precise as we need it to be, the explanatory use requires us to learn how the component parts of the target system give rise to the behaviour of the whole.

There is an additional explanatory use of models, one that is even more remote from the predictive case. Sometimes we want to understand how hypothetical systems 
work. There is really no analogue to this in the prediction case because in that context we are interested in predicting the behaviour of actual target systems. Sometimes in the course of our trying to explain actual target systems, we make comparisons to hypothetical systems. Fisher famously said that if we want to explain the prevalence of two sex organisms, we should start by explaining what the biological world would be like if there were three sexes [94].

As computational social science is a model-based field of science, aiming to both explain phenomena of interest and predict their evolution, we need to clearly understand the respective implications for model building.

\subsubsection{Qualitative analysis. Generative models}

Generative models are a third way of doing science, an escape from the deductive/inductive dichotomy. They allow qualitative analysis to be done in a rigorous and controllable way. A typical computational generative approach, as was proposed by Epstein, is agent-based simulation, requiring to:

"situate an initial population of autonomous heterogeneous agents (see also [50]) in a relevant special environment; allow them to interact according to simple local rules, and thereby generate - or "grow" - the macroscopic regularity from the bottom up" [95].

This passage raises some objections and requires that some caveats be taken into account.

First, how to find out the simple local rules? How to avoid ad hoc and aribitrary explanations? As already observed [96], one criterion has often been used, i.e., choose the conditions that are sufficient to generate a given effect. However, this leads to a great deal of alternative options, all of which are to some extent arbitrary. The construction of plausible generative models is a challenge for the new computational social science.

Second, the notion of rules needs clarification and revision. Possibly, rules should be replaced by explicit and theory-founded agent models, whch include not only decision-making mechanisms but also representations, attitudes, strategies, actions, motivations, and the like.

\subsubsection{Integrate heterogeneous models}

The analysis and modelling of large-scale social systems should be supported on three coordinated legs: collection, production and analysis of data; agent-based simulations; analytical modelling.

Well-motivated models of agents in interactions may generate unexpected collective effects that call for mathematical understanding. Conversely, collective effects predicted from models simple enough to be mathematically analyzed call for numerical tests on more complex (more realistic) multi-agents systems, or empirical validation through surveys or experiments, whenever this is possible. Modelling requires to find appropriate compromises between socio-physical realism, and mathematical simplicity.

With this general goal of understanding and anticipating behaviours of complex social systems, one needs to develop simplified models, on which the mathematical analysis can be done. Such models should be able to reproduce the stylized facts empirically observed. From the analysis of these models, new intuition can be gained, and more complex models can be studied, both numerically and analytically (see [97]). Scenarios can be explored and tested by the large-scale simulations, providing results that can be used for decision making. 
In the case of collective phenomena (crowds, pedestrian and auto traffic, fashion, financial or social crisis, opinion formation and epidemic propagation; see [81,98], the goal of modelling, with analytical and numerical means, is precisely to understand the global ('macroscopic') level from the characteristics of the constitutive elements (the 'microscopic' level) and the social structures they belong to, and also to understand how the collective level influences the individual behaviour. Such modelling can, and should, be done with multiple approaches, integrating tools and concepts from different disciplines: applied mathematics, statistical physics, computer science, theoretical economics (some relevant keywords would be: partial differential equations, optimal control theory, information theory, dynamical systems, statistical physics of disordered systems, graph theory, game theory, numerical simulations, agent-based systems), and this in close interaction and collaboration with social scientists.

At the interface between data analysis and modelling, the analysis of data may allow us to reveal robust statistical features that are characteristic of the system under study. A particular example is the empirical analysis of the network structure, in different contexts (networks of co-authorships, inter-bank network, road networks...). A stylized fact observed for a particular system (e.g. a small-world structure) becomes a guide for the modeller - either this particular network structure is an input to the model, or one of the goal of the model is to understand how such structure emerges. Developing new data analysis tools, as well as specific data representation and visualization tools, is thus an important component of the computational social approach.

\section{Expected impact}

\subsection{Impact on science}

The impact of progress along the above lines on science cannot be overstated. To begin with, sociology in particular and the social sciences in general would undergo a dramatic paradigm shift, arising from the incorporation of the scientific method of physical sciences. Thus, the combination of the computational approach with a sensible use of experiment will bring the social sciences closer to establishing a wellground link between theory and empirical facts and research. Such links should inform all sciences in which human behaviour is the main object of research or interest, and should solve incompatibilities such as economics relying on the rational actor picture and sociology and social psychology outright rejecting it; on the other hand, the latter rely much more on facts (identified from experiments, surveys, etc.) than traditional economics, based on the strength of purely abstract analytical approaches (for much more on this, see [99]). Computational social science would be a major factor toward this paradigm change in the social sciences.

On the other hand, the impact of the research we have described would certainly go beyond the social sciences, as it would provide new tools and methods that would be applicable in any instances where BigData are a key ingredient. As we expect that new analytical approaches will be developed along with algorithms and monitoring procedures for massive data, the complex systems perspective we propose here, in which entities and their interactions might be abstract and arise in any context, would immediately lead to applications of our findings in fields ranging from physics to ecology through systems biology. In fact, the complex systems approach that transpires through the entire proposal is a crucial lever towards blurring the dividing lines among disciplines and creating a truly interdisciplinary, non-compartmental science [100]. 


\subsection{Impact on technology and competitiveness}

Computational social science requires an enormous research effort on ICT, the pillar on which the research directions summarized here rely. Collecting, transmitting, analysing, simulating and incorporating data to simulations will require computational and communication capabilities well beyond the current state of the art facilities. While the EU research community on ICT is very active, a scientific program like the one discussed in this paper would boost it to take over the US and Japan advantage on this field, thus increasing the competitiveness of the EU as a result and advancing technology on a world level.

\subsection{Impact on society}

The role of computational social science is a leading one in addressing the BigProblems of society, avoiding crises and threats to its stability and healthy development.

Computational social science will provide many tools towards achieving this goal, and will play a leading role in the successful execution of the project. The development of Computational Social Science, as proposed in this manifesto, will make it possible to model and simulate social processes on a global scale, allowing us to take full account of the long distance interdependencies that characterise today's heavily interconnected world. The output of these simulations will be used to support policy makers in their decision making, to enable them to efficiently and effectively identify optimal paths for our society. Similarly, open access to these large scale simulations will support individuals in their evaluation of different policy options in the light of their personal needs and goals, greatly enhancing citizen participation in this decision process. These developments together open the doors to a much safer, more sustainable and fairer global society.

\section{Conclusions}

There is an increasing realization of the enormous potential of data-driven computational social science [9]. The availability of unprecedented amounts of data about human interactions in different social spheres or environments opens the possibility of using those data to leverage knowledge about social behaviour beyond research on the scale of tens of people. The data can be used to check and validate the results of simulation models and socio-economic theories, but a further step in using them is to take them into account already at the modelling stage.

At the same time, the relation between social simulation (e.g., agent based modelling) and theoretical understanding is still problematic. Thus, simulations must be accompanied by micro-macro-loop theories, i.e., theories of mechanisms at the individual level that affect the global behavior, and theories of loop-closing downward effects or second-order emergence.

In view of this, it is clear that naive or brute-force incorporation of large-scale data into simulation models may not lead to the expected results in terms of achieving relevant progress in social science. While it is apparent that the analysis of the data will certainly contribute to the understanding of mechanisms, it is also clear that further input will often be needed, in particular input obtained from experiments under controlled parameters or situations. These will shed light on the decision-making mechanisms that sometimes can be obscured among the midst of the data.

In conclusion, computational social science, as a rapidly developing and successful field, needs to be aware of the necessity to develop its theoretical premises, and to 
test them. Much as physical theories and models are tested through incredibly large experiments (such as the LHC at the CERN), progress in computational models of social phenomena will only be possible by a sensible combination of data input, experimental work, and theory devising. Computational social science requires brings along challenging demands on the experimental side, in terms of design and procedures, which can only be solved by working together with the computational science community.

The publication of this work was partially supported by the European Union's Seventh Framework Programme (FP7/2007-2013) under grant agreement No. 284709, a Coordination and Support Action in the Information and Communication Technologies activity area ('FuturICT' FET Flagship Pilot Project). We are grateful to the anonymous reviewers for the insightful comments.

\section{References}

1. D. Helbing, S. Balietti, Eur. Phys. J. Special Topics 195, 3 (2011)

2. Y. Song, L. Zhang, C.L. Giles, ACM Transactions on the Web 5 (2011)

3. D. Helbing, J. Jost, D. Lane, Adv. Complex Syst. 11, 485 (2008)

4. P. Richerson, R. Boyd, J. Social Biological Struct. 1, 127 (1978)

5. J.C. Chesnais, The Demographic Transition: Stages, Patterns, and Economic Implications: A Longitudinal Study of Sixty-Seven Countries Covering the Period 17201984 (Oxford University Press, 1993)

6. J.P. Onnela, J. Saramäki, J. Hyvonen, G. Szabó, D. Lazer, K. Kaski, J. Kertész, A.L. Barabási, PNAS 104, 7332 (2007)

7. D.V. Balcan, B. Colizza, H. Goncalves, J.J. Ramasco, A. Vespignani, PNAS USA 106, $21484(2009)$

8. M. Karsai, M. Kivelä, R.K. Pan, K. Kaski, J. Kertész, A.L. Barabási, J. Saramäki, Phys. Rev. E 83, 1 (2011)

9. D. Lazer, A. Pentland, L. Adamic, S. Aral, A. Barabási, D. Brewer, N. Christakis, N. Contractor, J. Fowler, M. Gutmann, T. Jebara, G. King, D. Roy, M.W. Macy, M. Van Alystyn, Science 323, 721 (2009)

10. T.C. Schelling, J. Math. Sociol. 1, 143 (1971)

11. L. Putterman, Science 328, 578 (2010)

12. D. Helbing, S. Bishop, R. Conte, P. Lukowicz, J.B. McCarthy, Eur. Phys. J. Special Topics 214, 11 (2012)

13. J. Henrich, J. Ensminger, R. McElreath, A. Barr, C. Barrett, A. Bolyanatz, J.C. Cardenas, M. Gurven, E. Gwako, N. Henrich, C. Lesorogol, F. Marlowe, D. Tracer, J. Ziker, Science 327, 1480 (2010)

14. H. Peyton Young, Social Norms and Norm Dynamics (Princeton University Press, 2009)

15. J.M. Epstein, Comput. Econom. 18, 9 (2001)

16. C. Bicchieri, The grammar of society: the nature and dynamics of social norms (Cambridge University Press, 2006)

17. E. Ullman-Margalit, Erkenntnis 11, 143 (1977)

18. A. Schotter, The Economic Theory of Social Institutions (Cambridge University Press, 2008)

19. J.M. Epstein, PNAS 99, 7243 (2002)

20. M. Mauss, The Gift: forms and functions of exchange in archaic societies (Routledge, London 1922)

21. J. Rouchier, M. O'Connor, F. Bousquet, J. Artificial Soc. Social Simulation 4 (2001)

22. A.S. Golder, B.A. Huberman, J. Information Sci. 32, 198 (2006)

23. C. Cattuto, V. Loreto, L. Pietronero, PNAS USA 104, 146 (2007) 
24. C. Cattuto, A. Barrat, A. Baldassarri, G. Schehr, V. Loreto, PNAS USA 106, 10511 (2009)

25. M.A. Nowak, K. Sigmund, Nature 393, 573 (1998)

26. K. Walker, Corporate Reputation Review 12, 357 (2010)

27. J.J. Ebbers, N.M. Wijnberg, Strategic Organization 8, 255 (2010)

28. R. Axelrod, The Evolution of Cooperation (Basic Books, New York, 1984), p. 1

29. W. Raub, J. Weesie, Amer. J. Sociol. 96, 626 (1990)

30. D. Vinković, A. Kirman, PNAS 103, 19261 (2006)

31. W.A. Clark, M. Fossett, PNAS USA 105, 4109 (2008)

32. L. Gauvin, J. Vannimenus, J.P. Nadal, Eur. Phys. J. B - Condensed Matter and Complex Systems 70, 293 (2009)

33. N. Gilbert, R. Conte (eds.), Artificial Societies: the Computer Simulation of Social Life (University College London Press, 1995)

34. N. Gilbert, R. Conte Artificial Societies: The Computer Simulation of Social Life (University College London Press, London, 1995), p. 144

35. D. Dennett, Times Literary Supplement 25, 3 (1995)

36. S. Havlin, D.Y. Kenett, E. Ben-Jacob, A. Bunde, R. Cohen, H. Hermann, J.W. Kantelhardt, J. Kertsz, S. Kirkpatrick, J. Kurths, Y. Portugali, S. Solomon, Eur. Phys. J. Special Topics 214, 273 (2012)

37. J.M. Pujol, A. Flache, J. Delgado, R. Sanguessa, J. Artificial Soc. Social Simulation 8, $41(2005)$

38. C. Alós-Ferrer, G. Kirchsteiger, Economic Theory 44, 339 (2006)

39. A. Yasutomi, Physica D: Nonlinear Phenomena 82, 180 (1995)

40. R.L. Axtell, J.M. Epstein, J.S. Dean, G.J. Gumerman, A.C. Swedlund, J. Harburger, S. Chakravarty, R. Hammond, J. Parker, M. Parkeraj, PNAS 4, 7275 (2002)

41. L.E. Cederman, Emergent Actors in World Politics: How States and Nations Develop (Princeton University Press, 1997)

42. R. Axelrod, Amer. Political Sci. Rev. 80, 1095 (1986)

43. S. Sen, S. Airiau, Proceedings of the 20th International Joint Conference on Artifical intelligence (ACM, Hyderabad, 2007), p. 1507

44. R. Hegselmann, O. Will, Proceedings of the 10th international conference on Multiagent-based simulation (Springer-Verlag, Berlin 2010), p. 123

45. D. Lewis, Convention. A Philosophical Study (Harvard University Press, 1969)

46. N.J. Saam, A. Harrer, J. Artificial Soc. Social Simulation 2 (1999)

47. M. Xenitidou, B. Edmonds (eds.), Dynamic View of Norms (Cambridge University Press, forthcoming)

48. R. Conte, G. Andrighetto, M. Campenn (eds.), Minding Norms (Oxford University Press, forthcoming)

49. W.B. Arthur, Handbook of Comput. Econom., 2: Agent-Based Comput. Econom. (Elsevier, 2005), p. 1551

50. W.B. Arthur, Amer. Econom. Rev. 84, 406 (1994)

51. M.W. Macy, A. Flache, PNAS USA 99, 7229 (2002)

52. J.M. Epstein, Generative Social Science: Studies in Agent-Based Computational Modeling (Princeton University Press, 2007)

53. J.M. Galán, L.R. Izquierdo, S.S. Izquierdo, J.I. Santos, R. del Olmo, A. López-Paredes, B. Edmonds, J. Artificial Soc. Social Simul. 12, 1 (2009)

54. N.A. Christakis, J.H. Fowler, N. Engl. J. Med. 357, 370 (2007)

55. R. Lyons, Stat. Politics Policy 2, 2 (2011)

56. T.A.B. Snijders, G.G. Van de Bunt, C.E.G. Steglich, Social Networks 32, 44 (2010)

57. P.L. Borrill, L. Testfatsion, The Elgar Companion to Recent Economic Methodology (Edward Elgar Publishers, New York, 2011), p. 228

58. F. Darema, Applications Systems, Proc. IEEE 93, 692 (2005)

59. Y. Benkler, Yale Law J. 112, 369 (2002)

60. D.C. Brabham, Convergence: The International Journal of Research into New Media Technologies 14, 75 (2008) 
61. A. Kittur, E.H. Chi, B. Suh, Proceedings of the twenty-sixth annual SIGCHI conference on Human factors in computing systems (ACM, New York, 2008), p. 453

62. N. Kiukkonen, J. Blom, O. Dousse, D. Gatica-Perez, J. Laurila, Proceedings of International Conference on Pervasive Services (ACM, Berlin, 2010)

63. N. Lane, E. Miluzzo, H. Lu, D. Peebles, T. Choudhury, A. Campbell, IEEE Communications 48, $140(2010)$

64. R. Conte, B. Edmonds, S. Moss, R.K. Sawyer, Comput. Math. Organization Theory 7, $183(2001)$

65. V.M. Eguíluz, M.G. Zimmermann, C.J. Cela-Conde, M. San Miguel, Amer. J. Scociol. 110, $977(2005)$

66. J.S. Sichman, R. Conte, R., Comput. Math. Organization Theory 8 (2002)

67. G. Ehrhardt, M. Marsili, F. Vega-Redondo, Phys. Rev. E 74, 1 (2006)

68. C. Castelfranchi, R. Conte, Microsimulation and the social science (Springer-Verlag, 1996), p. 394

69. G.P. Huber, Organization Science 2, 88 (1991)

70. G. Ritzer (ed.), Frontiers of Social Theory: The New Syntheses (Columbia University Press, 1990), p. 1

71. F.C. Billari, T. Fent, A. Prskawetz, J. Scheffran, Agent-Based Computational Modelling Applications in Demography, Social, Economic and Environmental Sciences (Physica Verlag-Springer, Heidelberg, 2006)

72. M. Assen, V. Buskens, R. Werner, J. Math. Sociol. 35 (2011)

73. R. Putnam, Bowling alone: the collapse and revival of American community (Simon \& Schuster, 2001)

74. H. Farrell, J. Knight, Politics \& Society 31, 537 (2003)

75. M. Shadnam, T.B. Lawrence, Business Ethics Quarterly 21, 379 (2011)

76. R. Axelrod, J. Conflict Resol. 41, 203 (1997)

77. D. Centola, J.C. González-Avella, V.M. Eguíluz, M. San Miguel, J. Conflict Resol. 51, 905 (2007)

78. A. Flache, M.W. Macy, J. Conflict Resol. 55, 970 (2011)

79. J.C. González-Avella, M.G. Cosenza, K. Klemm, V.M. Eguíluz, M. San Miguel, J. Artificial Social Simul. 10, 1 (2007)

80. X. Castello, F. Vazquez, V.M. Eguiluz, L. Loureiro-Porto, M. San Miguel, L. Chapel, G. Deffuant, Viability and Resilience of Complex Systems. Concepts, Methods and Case Studies from Ecology and Society (Springer Verlag, 2011), p. 49

81. D. Helbing, W. Yu, H. Rauhut, J. Math. Sociol. 35, 177 (2011)

82. J. Grujic, C. Fosco, L. Araujo, J.A. Cuesta, A. Sanchez, PLoS ONE 5, e13749 (2010)

83. W.S. Bainbridge, Science 317, 472 (2007)

84. L. Habib, W. Line, Mind, Culture Activity 14, 266 (2007)

85. R. Dunbar, Evolutionary Anthropology: Issues, News, and Rev. 6, 178 (1998)

86. R. Conte, M. Paolucci, Reputation in artificial societies. Social beliefs for social control (Kluwer, 2002)

87. R. Conte, M. Paolucci, J. Sabater Mir, Adv. Complex Syst. 11, 303 (2008)

88. F. Giardini, R. Conte, Simulation 88, 18 (2011)

89. M. Paolucci, T. Eymann, W. Jager, J. Sabater-Mir, R. Conte, S. Marmo, S. Picascia, W. Quattrociocchi, T. Balke, S. Koenig, T. Broekhuizen, D. Trampe, M. Tuk, I. Brito, I. Pinyol, D. Villatoro, Social Knowledge for e-Governance: Theory and Technology of Reputation (ISTC-CNR, Rome 2009)

90. R. Falcone, C. Castelfranchi, Trust Theory: A Socio-Cognitive and Computational Model (Wiley, 2010)

91. G.A. Akerlof, R.J. Shiller, Animal Spirits: How Human Psychology Drives the Economy, and Why It Matters for Global Capitalism (Princeron University Press, 2009), p. 1

92. W.C. Salmon, Four Decades of Scientific Explanation (Pittsburgh University Press, 1989)

93. M. Weisberg, When less is more: Tradeoffs and idealization in model building (Stanford University Press, 2003) 
94. R.A. Fisher, The Genetical Theory of Natural Selection (Clarendon, 1930)

95. J.M. Epstein, Complexity 4, 41 (1999)

96. F. Squazzoni (ed.), Epistemological Aspects of Computer Simulation in the Social Sciences (Springer, Berlin-Heidelberg, 2009), p. 29

97. D. Helbing, Sci. Culture 76, 315 (2010)

98. J. Lorenz, H. Rauhut, F. Schweitzer, D. Helbing, PNAS USA 108, 9020 (2011)

99. H. Gintis, The Bounds of Reason (Princeton University Press, 2009)

100. M. San Miguel, J. Johnson, J. Kertesz, K. Kaski, A. Daz-Guilera, R.S. MacKay, V. Loreto, P. Erdi, D. Helbing, Eur. Phys. J. Special Topics 214, 245 (2012) 